\title{
3-year-old children's memory flexibility allows adaptation to an altered context
}

\author{
Krisztina Liszkai-Peres ${ }^{a, b, c}$, , Dora Kampis ${ }^{d}$, Ildikó Királye,f
}

\section{[preprint before peer review]}

Author affiliation:

${ }^{a}$ Department of Ethology, Eötvös Loránd University Budapest, Hungary

bDoctoral School of Psychology, Eötvös Loránd University, Budapest, Hungary

'Institute of Psychology, Eötvös Loránd University, Budapest, Hungary

dUniversity of Copenhagen, Copenhagen, Denmark

eMTA-ELTE Momentum Social Minds Research Group, Psychology Institute, ELTE

Eötvös Loránd University, Budapest, Hungary

fCognitive Development Center, Central European University, Budapest, Hungary

\section{Acknowledgments:}

The project was supported by the National Science Fund (OTKA 116779 ) and by the Momentum Program of the Hungarian Academy of Sciences (LP-2017-17/2017, PI:

Ildikó Király).

Conflict of interest:

The authors declare no conflict of interest.

Corresponding author:

Krisztina Liszkai-Peres: peres.krisztina@ppk.elte.hu

The data that support the findings of this study are openly available in Open Science Foundation at: https://osf.io/jx4q6/?view_only=ffd5fb77c46c4729bce5b41d6077f38e 
Running Head: 3-year-old children's memory flexibility allows adaptation to an altered context

\section{Research Highlights}

- 3-year-olds selectively imitated tool use based on its efficacy in an immediate imitation test.

- After a week delay, relevance of the tool changed from irrelevant to relevant and vice versa.

- 3-year-olds changed their means towards a goal and used the tool within the new constraints where it became relevant.

- However, children did not fully omit using the tool within the new constraints where it lost its efficacy.

- 3-year-olds flexibly retrieve an action from memory if needed; otherwise they tend to use their former strategy due to cultural learning. 
Running Head: 3-year-old children's memory flexibility allows adaptation to an altered context

\section{Abstract}

Imitation provides a reliable method to investigate the developing memory functions in childhood. The present study explored whether 3-year-old children are able to revise their previous experiences after a 1 week delay in order to adapt to an altered context. We used a combined immediate (Session 1) and delayed (Session 2) imitation paradigm. The constraints (target object close/far) and relatedly the relevance of using a tool in a goal attainment task (irrelevant/relevant, respectively) changed between the sessions. We found that children in Session 1 used the tool only when it was needed (relevant/object far context). After the 1 week delay when the tool was previously irrelevant and then became relevant, children remembered the irrelevant act and applied it in the altered context. When the tool lost its relevance after 1 week, children used the tool less than before, but did not fully omit it, despite its reduced efficiency. We propose that the flexible restoration of a formerly irrelevant act and the maintenance of a formerly successful solution indicate flexibility of children's memory when guiding imitation. This flexibility, however, interacts with children's tendency to remain faithful to strategies that were previously ostensively demonstrated to them.

Keywords: memory flexibility, constraint change, delayed imitation, updating, episodic memory 
Running Head: 3-year-old children's memory flexibility allows adaptation to an altered context

\section{Introduction}

In early childhood, imitation i.e., behavioral re-enactment is a very important means for learning, both for instrumental purposes such as using a tool, and also for socially constituted forms of behavior, like gestures and even language (Meltzoff \& Marshall, 2018; Meltzoff \& Williamson, 2013). Already during the first year of life, infants are able to copy simple facial gestures and vocalizations (Meltzoff \& Moore, 1983). With the development of the motor system, more complex behavior sequences are also imitated (for a review see Jones, 2009), and with age the length of the imitated sequence is constantly growing. Precision of imitation shows variability from high fidelity imitation, copying each action step, to goal emulation, when only the goal is copied, but the specific means leading to it are not (Bekkering, Wohlschläger, \& Gattis, 2000; Carpenter, Call, \& Tomasello, 2005; Gergely, Bekkering, \& Király, 2002; Nielsen \& Blank, 2011). This variability in the form of re-enactment highlights that the underlying mechanism is likely not a single direct copying process (Paulus, 2014), but rather a manifold one that is inferential in nature (Meltzoff, 1995b).

The notion that imitation is inferential is supported by the fact that goals play a modulatory role in what gets re-enacted. In the seminal paradigm developed by Meltzoff (1995), for example, children typically do not blindly copy a model's unsuccessful action (a failed attempt); instead they imitate the model's inferred goal with successful goal-attainment. Children are also found to be selective in imitating a tool use action, as they are more prone to imitate if the successful goal-attainment with a tool is preceded by another inefficient means (Nielsen, 2006; Want \& Harris, 2001). In addition, already at a young age, children copy only those specific aspects of an 
Running Head: 3-year-old children's memory flexibility allows adaptation to an altered context

event that they deem efficient in a given context (Gergely et al., 2002). In light of these results, the teleological account suggests that young children already apply the presumption that a goal is attained in the most efficient way possible within the constraints of a situation, and use this assumption in their choice of what to re-enact from the observed behavior - that is, they infer the intended goal of the action (Gergely \& Csibra, 2003). Though this inference-based explanation of imitation has been challenged by low-level accounts of action interpretation (e.g. Juvrud \& Gredebäck, 2020; Paulus, 2014; Ray \& Heyes, 2011), this conceptual approach could offer a viable solution for learning completely novel behaviors through goal identification and a related initiation of a search for a means to attain it (see Király \& Oláh, 2020).

The inference-based nature of imitation makes it an excellent means to study the development of learning and memory in more depth (Jones \& Herbert, 2006; Meltzoff, 1988), by probing the understanding of causal relations and what gets retained from different events. Do irrelevant actions get filtered out? Do efficiency evaluations play a role in what is remembered? Memory studies applying delayed imitation paradigms, where some time passes between the demonstration of an action and the recall of this action (varying from 1 day to months), have mainly focused on imitation as a behavioral mode of free recall, and probed the changes of the reliability and the capacity constraints of memory across development (e.g., Bauer \& Leventon, 2013; for a review see Jones \& Herbert, 2006; Meltzoff \& Williamson, 2013). These studies do not examine, however, memory encoding and retrieval separately. We argue that these two should be investigated separately to better understand what determines whether information is retained or filtered out at encoding, what part of said encoded information is retrieved after a delay, and what is filtered out at retrieval (or failed to be retrieved). 
Running Head: 3-year-old children's memory flexibility allows adaptation to an altered context

Memory is a dynamic system, working in interaction with different aspects of the environment. For example, socio-communicative cues are crucial already at event encoding as infants encode more information in an ostensive, social context than in a non-communicative situation (Brugger, Lariviere, Mumme, \& Bushnell, 2007; Király, Csibra, \& Gergely, 2013; Nielsen, 2006). Social cues are also important in the retrieval phase, as reproduction of a formerly demonstrated act is influenced by the presence of the model during the test situation (Király, 2009; Marsh, Ropar, \& Hamilton, 2019; Stengelin, Hepach, \& Haun, 2019).

Memory is also dynamic in the sense that the same memory often has to be retrieved in various contexts. Events in real life are often similar but not identical, and the solution to a previous problem can be transferred to another one with some correction, fitting to the new constraints. For example, if we have driven only a car with manual transmission, and then we have to drive a car with automatic transmission, we are still able to drive the car, but we have to tailor our behavior to the new circumstances. Evidence indicates that some foundations of such adjustment can be observed early on, as already at a preschool age children can modify their behavior in simple scenarios and adapt to new constraints (Williamson \& Meltzoff, 2011; Williamson, Meltzoff, \& Markman, 2008). For example, in the study of Williamson et al. (2008) three-year-olds had either an easy or a difficult prior experience with opening a puzzle box. Children imitated a new opening method less if their own means had been efficient (easy opening context) compared to children with a difficult prior experience. Subsequently, if it became difficult to open the box, children were able to retrieve the demonstrated act and use it instead of their now less efficient means (Williamson et al., 2008). Thus, children were able to adjust their behavior relative to a past event 
Running Head: 3-year-old children's memory flexibility allows adaptation to an altered context

(i.e., choose a different means), based on the evaluation of which means can most efficiently reach the goal.

Other times we have to adjust our present behavior in relation to a possible future event. In tasks involving episodic foresight, children have to prepare and overcome a possible future obstacle based on their previous experiences (Atance \& Sommerville, 2014; Scarf, Gross, Colombo, \& Hayne, 2013; Suddendorf, Nielsen, \& Von Gehlen, 2011). For example, in the study of Scarf et al. (2013) preschoolers found a locked treasure chest in a sandbox. One day later, before visiting the sandbox again, they were allowed to choose from various objects including a key. If children could connect the memory of the locked treasure box and the future scenario of meeting it again, they would choose the key. Four-year-olds were able to solve the task, but 3-year-olds only succeeded when the delay between the past and future event was no more than 15 minutes (Scarf et al., 2013). In a similar vein, Blankenship \& Kibbe (2022) found that even two-year-olds show flexibility in a simpler future oriented task; and 3- and 4-yearold children could solve a multiple-step search problem with specific goals by recruiting both the retrieval of recent episodes from their working memory and their planning abilities, though 4-year-olds performed better on longer event-sequences than 3-yearolds (Blankenship \& Kibbe, 2019). Based on these results, flexibility of memory may be restricted to shorter intervals around the second and third year of life, yet these studies already show evidence of the dynamic interaction of contextual demands and memory retrieval (Blankenship \& Kibbe, 2019, 2022; Scarf et al., 2013).

The abovementioned studies (Blankenship \& Kibbe, 2019; Scarf et al., 2013; Williamson et al., 2008) share that the retrieval of relevant memories helped children to successfully attain a goal. However, in said studies the goal situation (with the modified context) and the prior experience (the source of their memories) were close 
Running Head: 3-year-old children's memory flexibility allows adaptation to an altered context

in time. Consequently, it remains unclear how this kind of flexibility works if the delay is longer than the interval with which short-term memory operates. Studying these processes with a longer delay between encoding and retrieval is essential to investigate what is consolidated and stored for the long-term in memory, and whether these memory traces are available for flexible adjustment in behavior.

Some studies explored whether after a longer delay the content of memories changes if retrieved by behavioral re-enactment (Kline, Gervais, Moya, \& Boyd, 2020; Simpson \& Riggs, 2011). In Simpson and Riggs' (2011) study, 3- and 4-year-old children imitated both irrelevant and relevant acts in the short-term, but after a week delay children mainly preformed only the relevant actions in order to achieve a goal. In a cross-cultural study by Kline et al. (2020), children (between the age of 9 and 16 years) and adults imitated more precisely on the short-term, but omitted irrelevant actions after a one month delay. As Kline et al. (2020) argued, impact of social motivations could have been stronger during the first session, as imitation took place within an interaction between the demonstrator and the participant. One month later the demonstration part was missing, which could have weakened the influence of social factors and facilitated a more rational solution. An alternative explanation would be that at the immediate recall all the elements were available in working memory and thus were retrieved, while for the long-term only the meaningful elements were stored and later available for retrieval. Furthermore, it is not clear whether the omission of the irrelevant actions is only a response to the situational demands - i.e., thus the selective re-enactment is an efficient, adaptive solution while the irrelevant acts could be still retrieved if needed - or whether memory holds only relevant action elements in the long-term. 
Running Head: 3-year-old children's memory flexibility allows adaptation to an altered context

There are broadly two types of changes that may take place affecting the relevance of particular memory elements, which may require slightly different types of flexibility and adjustment. One type of change is that an element or act encoded as relevant becomes irrelevant in a context of retrieval. In this case the current task that imposed the retrieval demand could still be solved with the previous means, but it is less efficient within the changed constraints. Hence, adaptation could be achieved through an update of the previous means' relevance, (and consequently in the retrieval of the stored memory), within the modified constraints. The behavior thus could reflect two operations. First, the original episode could be retrieved and updated in a way to adapt to the situation at hand, and thus result in the omission of event element that became 'irrelevant'. However, there is an alternative route, in which there is no need to recall the entire action sequence, just the relevant ones, in search for the easiest way of attaining the goal. Overall, the behavioral outcome in itself cannot prove nor exclude the potential availability of all the action elements in memory. However, reenactment of the less efficient action may reflect that the updating of memory has not taken place. The other direction is that a previously irrelevant act or element of the observed behavior becomes relevant within new constraints. In this case recall and reevaluation of the previously irrelevant act is essential as the task could not be solved without it.

Considering these possibilities can help answer the question of what explains the omission of an irrelevant action element after a longer delay (see Kline et al. (2020); Simpson \& Riggs (2011)). One solution is to introduce a challenge in the form of a problem that could only be solved with the help of retrieving the entire original episode, including the previously irrelevant, but within the present constraints necessary, element as well. A previous study has investigated whether the two types of constraint 
Running Head: 3-year-old children's memory flexibility allows adaptation to an altered context

changes described above induce flexible adaptation in recall in 2-year-olds (LiszkaiPeres, Kampis, \& Király, 2021). In a delayed imitation paradigm, relevance of a tool used for goal attainment changed between sessions (1 week delay). Infants were able to partially update their strategy to attain a goal by omitting a previously relevant action that has lost its relevance in the novel context (under new constraints).

Critically, 2-year-olds were less flexible when they had to recall a previously irrelevant action, which gained relevance within the changed constraints. Based on the results it was supposed that imitative flexibility at the age of two is emerging and is still limited. Children readily updated when it simply required omitting a previously used action, but retrospectively recollecting and reconsidering an act caused difficulties. As an additional follow-up study showed, recall of the irrelevant action decreased even immediately after the demonstration, indicating that at this age the causally relevant actions are encoded selectively and preserved for the long-term. This raises the question of when children would show fully flexible updating of their actions by recalling and re-enacting a previously irrelevant action step. Such an update requires episodic recollection by recalling the original causal analysis of the scene during encoding that led to the omission of that step, re-evaluating the current action steps, and finally incorporating the previously omitted step now as an efficient (and necessary) means to attain a goal.

There is some evidence indicating that at three years of age children can update their representations based on re-evaluating past events that involves episodic memory (Király, Oláh, Csibra, \& Kovács, 2018). In line with this, in Liszkai-Peres, Kampis et al. (2021) at the age of two some children were able to update their means, though this was not the dominant behavior at that age. Based on these studies, 3-yearolds are an ideal group to probe updating in a goal attainment situation with changed 
Running Head: 3-year-old children's memory flexibility allows adaptation to an altered context

contextual constraints. The flexibility of memory is still a developing skill at the age of three, and based on episodic foresight studies it works only for shorter intervals (Suddendorf et al., 2011). However, episodic foresight includes planning as well which might put a further load on children (Blankenship \& Kibbe, 2019). Scenarios that focus only on past events (Liszkai-Peres, Kampis et al., 2021) might be a less demanding task as opposed to the episodic foresight tasks and thus may be more suitable for probing memory flexibility in children. Based on this, we could expect that 3-year-old children would evaluate the relevance of action steps flexibly both at encoding and (delayed) retrieval and re-enact the necessary actions in an imitation scenario exactly. At the same time, around 3 years of age another phenomenon emerges. From this age onward children tend to copy both relevant and even perceivably irrelevant actions in relation to a given goal more faithfully than younger children (Nielsen, 2006; Over \& Carpenter, 2012). This phenomenon, termed over-imitation, is governed by both cognitive and motivational processes, but mainly rooted in the development of normative action parsing and the growing motivation for affiliation (Hoehl et al., 2019). The pedagogical setting of a situation, in which the model communicates with ostensive-referential cues, contributes to this effect (Csibra \& Gergely, 2009). In addition, from very early on, children rely on the demonstration of adults about the function of artifacts in order to narrow down their possible usage (Casler \& Kelemen, 2005). Based on these, if the old means still works and thus there is no trigger to induce the reevaluation of previous actions, 3-year-old children might stick to a former solution they observed in a communicative scenario, instead of focusing on the efficiency of the means within the new context. Therefore 3-year-old children might be less selective than 2-year-olds especially in the second session, when a prior relevant act that was 
Running Head: 3-year-old children's memory flexibility allows adaptation to an altered context

demonstrated to them ostensively becomes irrelevant. In such cases, they may stick to the previously successful strategy more than the 2-year-olds.

In the present study we investigated whether 3-year-olds can accommodate their behavior to the changing situational requirements after a 1 week delay. In the Irrelevant-Relevant condition in Session 1 the tool use was irrelevant, and it gained relevance in Session 2. In the Relevant-Irrelevant condition the constraints were reversed: in Session 1 the tool use was relevant, and in Session 2 it lost its relevance. Additionally, a baseline condition was designed to measure frequency of tool use without demonstration in the Relevant constraint (Baseline), and a control condition to measure the effect of a constraint change in the short-term, where demonstration in the Irrelevant constraint was followed immediately by the Relevant test (IrrelevantRelevant Immediate).

\section{Method}

The experimental design, materials and procedure are based on a previous study with 2-year-olds (Liszkai-Peres, Kampis et al., 2021).

\section{Participants}

The participants of this study were $\mathrm{N}=66$ preschoolers living in a large capital city in Europe. Children's ages ranged from 33 to 54 months, with a mean of 43.5 months ( $S D=4.1$ month). A further 18 children were tested but excluded from all analyses due to passivity (6), fussiness (3), experimenter error (4), or participation only in one test instead of two in conditions requiring two test occasions (5). In the final sample, 18 children (8 males) were in the Irrelevant-Relevant condition, 18 children (12 males) in the Relevant-Irrelevant condition, 12 children (4 males) in the Relevant 
Running Head: 3-year-old children's memory flexibility allows adaptation to an altered context

Baseline condition, and 18 children (8 males) in the Irrelevant-Relevant Immediate condition.

The study was approved by the Research Ethics Committee of the University. The parents of all participating children gave written informed consent before participation. Children were given a small gift for their participation.

\section{Materials}

The object set contained three toys with removable parts and a white wooden spoon (See Figure 1, and Supplemental Information Figure 1). Each toy had two separable parts, neither of which could fulfill the toy's function alone. There was a plastic propeller with a green head part $(20 \mathrm{~cm}$ long) that could be detached from an orange stick (19 cm long), and after joining the parts, the propeller could be whirled. Another object was an orange polystyrene ball $(4.5 \mathrm{~cm}$ diameter) cut into half, with hidden magnets. Once the halves stuck together, the ball could be thrown by one hand and caught by the other like a regular ball. The third object was a bicycle horn $(16 \mathrm{~cm}$ long) consisting of a plastic tube $(9 \mathrm{~cm}$ long) and a rubber end $(7 \mathrm{~cm}$ long). After assembling the horn, the rubber end could be squeezed to make a loud sound.

Demonstration and tests were completed on a table $(76 \times 106 \times 52 \mathrm{~cm})$. The separated parts of the toys were placed in a detached form onto one of two white rectangular panels (long panel $74.5 \times 29 \mathrm{~cm}$; short panel $28 \times 29 \mathrm{~cm}$ ) on a black tablecloth $(145 \times 200 \mathrm{~cm})$. One part of the toy was always as close to the participants as possible, the other part was always further away, either within arm's reach for the child (ca. $30 \mathrm{~cm}$ ) (irrelevant constraints for tool use) or out of reach (ca. $70 \mathrm{~cm}$ ) (relevant constraints for tool use). Distance was modified according to the abilities of the children 
Running Head: 3-year-old children's memory flexibility allows adaptation to an altered context

(e.g. for a tall, long-handed child the object was placed further away). A wooden spoon (30.5 $\mathrm{cm}$ long) was placed on the right side of the table as an aid for reaching target objects (Figure 1).

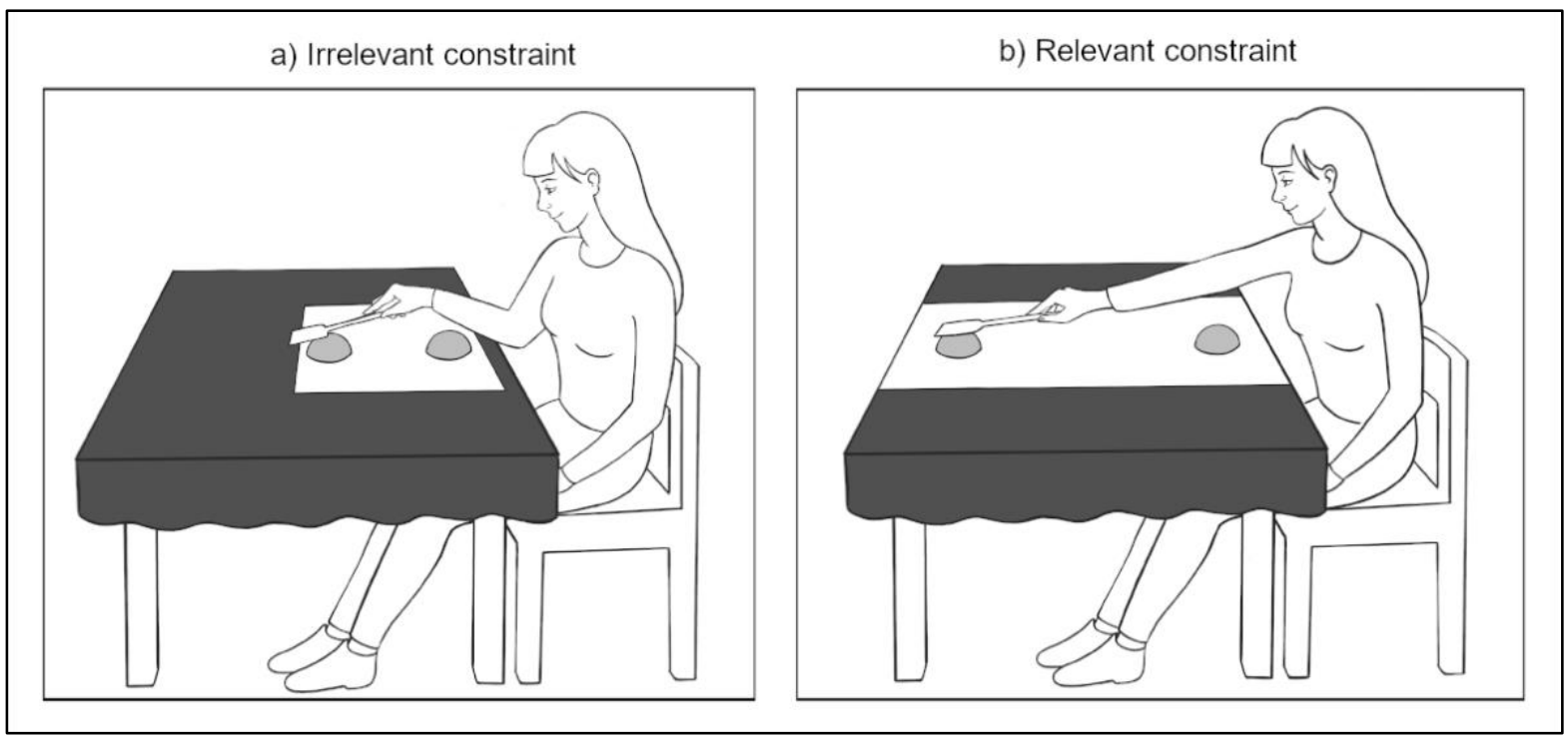

Figure 1. Irrelevant and Relevant constraints during demonstration phase. One part of the objects was always close to the experimenter/participant; the goal was to collect the other object part. In the irrelevant context the other part of each pair was within arm's reach, thus the situational constraints made the tool use unnecessary. In the relevant context the second part was further away and could not be reached by hand, thus tool use was necessary.

\section{Design and procedure}

The study included three experimental conditions and a baseline condition. Each experimental condition started with a demonstration, which was followed by an immediate imitation test. Two experimental conditions also included an additional deferred imitation test with a changed context, which was carried out one week after the first session (for a detailed description of the design see Figure 2). The third 
Running Head: 3-year-old children's memory flexibility allows adaptation to an altered context

experimental condition only involved the immediate re-enactment in a changed context. In the baseline condition, there was no demonstration of tool use (see below). Children were randomly assigned to a condition.

Irrelevant-Relevant and Relevant-Irrelevant conditions

The experiments were conducted by one of three experimenters (two females and a male). In both conditions two sessions were carried out. While Session 1 consisted of a demonstration phase and an immediate reenactment test, Session 2 occurred one week later and only included a delayed re-enactment test. Children sat on the caregiver's lap, next to the experimenter. The props were put on the table out of reach for the child, and parents were also asked to prevent children from touching them during demonstration. The experimenter sat in front of the white panel of the table on which each of the toys sets were placed earlier, their parts separated.

In the demonstration, the experimenter pointed to the part of the set at the closer end of the table saying, "Look, this is one part of the toy!", then pointed at the other part of the set placed further saying, "Look, there is the other part of it!". If the other part was close to her (within arm's reach) she grabbed the wooden spoon, showed it to the child, and reached the target object with it. If the other part was out of arm's reach, to emphasize distance of the target object, she first tried to reach it by stretching her hand as far as she could. After recognizing her unsuccessful action (“Uh-oh”), she grabbed the wooden spoon and got hold of the target object with it. Then she assembled the two parts, showed the function of the toy (e.g., the propeller whirling, throwing and catching the ball, the horn making a sound) and then put it away under the table. She demonstrated the assembly of the remaining two objects with the same 
Running Head: 3-year-old children's memory flexibility allows adaptation to an altered context

procedure. The toys were presented in two predefined orders (order A: propeller, ball, horn; order B: horn, propeller, ball), and the part children needed to retrieve was the same for each child: the head of the propeller, the rubber end of the horn, and one part of the ball (the ball's two parts were identical).

An immediate test followed the demonstration. At the beginning of the test the experimenter and the participant switched places. Then one of the object sets was placed (in detached form) onto the cleared table in the same position as it was during the demonstration (close/far), and the child was encouraged to play with it ("Now, you can play with it!"). If the child did not initiate any action, the experimenter tried to call the child's attention to the object part by pointing and saying, "Look, there is the other part of it!". After assembling one toy, the experimenter hid it under the table and put another object set onto the table. Children received the toys in the same order as they were demonstrated (order $\mathrm{A}$ or $\mathrm{B}$, see above). In the relevant tool use context, if participants tried to attain the distant part only with their hands, after 1 minute (or if the child lost interest in obtaining the object) the experimenter took the current set away and placed the next set on the table (this happened in $3.5 \%$ of the trials in the relevant context (7 out of the 198 relevant context trials)) (see also Supplemental Information Table 1 for details on success in tool use). If children used the tool in a subsequent trial, the experimenter placed the previously unsuccessful trial's object set in the original distant position $(1.5 \%-5$ out of the 198 relevant context trials). Successful tool use in these additional trials were counted into the total number of tool use trials for that child. If children did not use the tool in any of the three trials, after the third trial both of the object parts for each set were placed within arm's reach of the child, to assess whether children would assemble the sets $(1 \%-2$ out of the 198 relevant 
Running Head: 3-year-old children's memory flexibility allows adaptation to an altered context

context trials). No such intervention was carried out in the irrelevant context, as children could easily attain the toys.

Session 2 was conducted one week later and the child had to re-enact, without prior demonstration, the actions presented one week earlier. The task remained the same as before: to retrieve the second part and assemble the objects. The difference between the two sessions was that in the Irrelevant-Relevant condition in Session 2 the target objects were further away than earlier (making the tool use necessary), and in the Relevant-Irrelevant condition in Session 2 the objects were closer than they were in the previous session (making the tool use suboptimal, unnecessary).

Irrelevant-Relevant Immediate condition

In the Irrelevant-Relevant Immediate condition only one session was conducted, where immediately after the demonstration in the irrelevant context the constraints were modified so as to make tool use relevant and children had to execute the task in this more effortful situation.

\section{Relevant Baseline}

The Relevant Baseline only included one session. The test was carried out with the same object set and tool, but there was no demonstration of reaching with the tool or demonstration of assembling the objects. However, the experimenter indicated that the two parts belong together by showing the object's function in attached form (e.g., whirling, throwing, making sound) before taking it apart and putting the parts onto the table. To make the use of the object relevant, the target part was out of the children's reach. The instruction was, "Let's play with them!". If children were passive for a long 
Running Head: 3-year-old children's memory flexibility allows adaptation to an altered context

time, the experimenter pointed to the other piece saying, "This is the other part of the toy, let's play with it!", to motivate the child to retrieve the object.

\begin{tabular}{|l|c|c|c|}
\hline \multirow{2}{*}{ Conditions } & \multicolumn{2}{|c|}{ Session 1 } & $\begin{array}{c}\text { Session 2 } \\
\text { (1 week delay) }\end{array}$ \\
\cline { 2 - 4 } & $\begin{array}{c}\text { Demonstration } \\
\text { (model) }\end{array}$ & $\begin{array}{c}\text { Immediate } \\
\text { re-enactment (child) }\end{array}$ & $\begin{array}{c}\text { Delayed } \\
\text { re-enactment (child) }\end{array}$ \\
\hline Irrelevant - Relevant & Irrelevant & Irrelevant & Relevant \\
\hline Relevant - Irrelevant & Relevant & Relevant & - \\
\hline $\begin{array}{l}\text { Irrelevant - Relevant } \\
\text { Immediate }\end{array}$ & Irrelevant & Relevant & - \\
\hline Relevant Baseline & - & Relevant & - \\
\hline
\end{tabular}

Figure 2. The figure shows the experimental design. Conditions could consist of three phases: demonstration, immediate re-enactment, and delayed re-enactment. The demonstration and immediate re-enactment took place at the same occasion, in Session 1. One week later, in Session 2 only the delayed re-enactment took place. Relevance of tool use (relevant/irrelevant) changed between or within sessions according to the conditions.

\section{Coding}

Test videos were coded off-line. For each trial, children were given a score of 1 if they used the tool (target act), thus the total number of tool uses (dependent variable) ranged from 0 to 3. A behavior was coded as "tool use" if the participant grabbed the tool and reached with it toward the second object part regardless of whether this movement was successful or not (see also Supplemental Information, Table 1 on the success of tool use). In $98 \%$ of the trials coded as tool use, preschoolers were able to 
Running Head: 3-year-old children's memory flexibility allows adaptation to an altered context

attain the object with the tool and in only $2 \%$ of the trials were their attempts to reach the object with the tool unsuccessful. Only those participants who reached toward the object part either by hand or with the tool were included in the dataset. As such, behaviour was coded as "lack of tool use" if children used their hands for obtaining the object. Children were excluded from the dataset if they did not try to obtain the toys ("passive"), did not pay attention during demonstration, refused to play altogether, or

quit the test session before completing it ("fussy") (see Participants section). Joining object parts after obtaining both pieces was not an inclusion criterion, but children successfully assembled the object sets in $99 \%$ of the trials, and in $76 \%$ even without a prompt (see details in Supplemental Information, Figure 2-5). Generally, assembling the toys was easy for children, given that this procedure was unsuccessful in only $1 \%$ of all the trials. The children either requested help ( 2 trials out of a total of 5 unsuccessful trials), lost interest (2 trials out of a total of 5 unsuccessful trials) or did not know what to do (1 trial out of a total of 5 unsuccessful trials).

Inter-coder reliability was high in the Irrelevant-Relevant and Relevant-Irrelevant conditions (Cohen's kappa $=.94, p<.001$ ). Another independent coder coded $50 \%$ of the Irrelevant-Relevant Immediate videos and $50 \%$ of the Baseline videos. Reliability between coders was good (Cohen's kappa $=.93, p<.001)$.

\section{Results}

SPSS 25.0 for Windows was used for statistical analysis, and $p<.05$ was accepted as significant throughout. The dependent variable was whether children performed the target action (tool use). For the Generalized Linear Mixed Model (GLMM) analyses we included trials as a random factor, therefore the analyses were 
Running Head: 3-year-old children's memory flexibility allows adaptation to an altered context

executed on the scores children received for each trial (0 or 1$)$, while in all other analyses the sum of trial scores (0-3) was used.

GLMM with binary regression was used to test whether tool use can be explained by Condition (Irrelevant-Relevant, Relevant-Irrelevant, Irrelevant-Relevant Immediate, Relevant Baseline), Session (1, 2), Trial (1, 2, 3), Sex (male, female), Age, Object order (A, B) and Experimenter (Experimenter 1, 2, 3). The initial model included main effects and the interaction of Condition and Session. We used backward elimination for model selection. Then, we used planned comparisons to explain the model further.

The GLMM showed Condition x Session interaction $F(1,300)=51.28, p<.001$, whereby children used the tool differently between sessions depending on the condition (which differed by constraint change).

To assess the changes in tool use between sessions, we compared Session 1 and Session 2 in the two experimental conditions separately, with a Wilcoxon Signed Rank test. In the Irrelevant-Relevant condition children used the tool in Session 2 $($ Mean $=2.72, S D=0.75)$ significantly more frequently than in Session $1($ Mean $=0.17$ $S D=0.38), Z=-3.82, p<.01, r=-0.63$. In contrast, in the Relevant-Irrelevant condition children used the tool significantly less often in Session $2($ Mean $=1.94, S D=1.26)$ compared to Session $1($ Mean $=2.78, S D=0.73), Z=-2.57, p=.01, r=-0.43$ (Figure 3). In light of these results, children reacted to the constraint changes in both conditions: the frequency of their tool use differed significantly between Session 1 and 2.

Next, we probed whether within the two sessions children's propensity for tool use differed depending on the condition (and thus as a function of the tool's relevance). 
Running Head: 3-year-old children's memory flexibility allows adaptation to an altered context

With a Kruskal-Wallis test we compared conditions within Session 1 and 2 separately. In the analysis of Session 1 we used tool use as the dependent variable and condition as a between-subjects variable (Irrelevant-Relevant S1, Relevant-Irrelevant S1, Irrelevant-Relevant Immediate S1, Relevant Baseline S1). In Session 1, conditions differed significantly in tool use, $H(3)=43.07, p<.001, \eta^{2}=.65$. Dunn's pairwise tests with Bonferroni correction revealed that Relevant Baseline (Mean $=1.17, S D=1.4$ ) differed from the conditions where tool use was relevant (Relevant-Irrelevant (Mean = 2.78, $S D=0.73), p=.019 ;$ Irrelevant-Relevant Immediate $($ Mean $=2.83, S D=0.71)$, $p=.011$ ), but not from the condition where the tool use was irrelevant in this first session (Irrelevant-Relevant (Mean $=0.17, S D=0.38), p=.346)$. Moreover, in Session 1 the condition in which tool use was irrelevant (Irrelevant-Relevant, Mean $=0.17, S D$ $=0.38$ ) differed from the conditions in which it was relevant (compared to RelevantIrrelevant $($ Mean $=2.78, S D=0.73): p<.001 ;$ Irrelevant-Relevant Immediate $($ Mean $=$ 2.83, $S D=0.71): p<.001$ ) (Figure 3). Thus, in Session 1 children used a tool significantly more in the conditions where it was relevant for goal attainment and was demonstrated before, than if the tool use was not relevant or had not been demonstrated.

For analyzing Session 2, we compared conditions with a Kruskal-Wallis test with tool use as the dependent variable and condition as a between-subjects variable (Irrelevant-Relevant S2, Relevant-Irrelevant S2, Relevant Baseline S1), and likewise it showed that conditions differed in tool use, $H(2)=9.95, p=.007, \eta^{2}=.18$. Post-hoc analyses with Bonferroni correction indicated that Relevant Baseline (Mean $=1.17, S D$ $=1.4$ ) differed from the condition in which tool use was relevant in Session 2 (IrrelevantRelevant $($ Mean $=2.72, S D=0.75), p=.012)$, but not from the condition in which it 
Running Head: 3-year-old children's memory flexibility allows adaptation to an altered context

was irrelevant (Relevant-Irrelevant $($ Mean $=1.94, S D=1.26), p=1)$. Comparing the two experimental conditions, the second session of the Relevant-Irrelevant (Mean $=$ $1.94, S D=1.26)$ and the Irrelevant-Relevant $($ Mean $=2.72, S D=0.75)$ conditions did not differ significantly from each other, $p=.321$ (Figure 3). These results suggest that in Session 2, children used the tool more often if it was demonstrated to them before in the condition where the tool use became relevant, compared to when it was not demonstrated. However, while they were able to retrieve the tool use from memory when the constraint change proved it to be relevant, children still used the tool in the situation when it lost its relevance with comparable frequency. To confirm this claim we compared tool use in the irrelevant context of the two experimental conditions (Session 1 of Irrelevant-Relevant and Session 2 of Relevant-Irrelevant). A MannWhitney $U$ test showed difference $U=45, p<.001, \eta^{2}=0.47$, children used the tool more often in the irrelevant context during Session $2($ Mean $=1.94, S D=1.26)$ than during Session $1($ Mean $=0.17, S D=0.38)$.

Finally, with a Mann-Whitney test we analyzed whether there is a difference between constraint changes that happen after a delay or immediately after demonstration in the irrelevant tool use context. Comparison of Irrelevant-Relevant $($ Mean $=2.72, S D=0.75)$ and Irrelevant-Relevant Immediate $($ Mean $=2.83, S D=0.71)$ conditions showed that timing of constraint change did not influence tool use imitation, $U=145, p=.324, \mathrm{n}^{2}=.01$ (Figure 3). 
Running Head: 3-year-old children's memory flexibility allows adaptation to an altered context

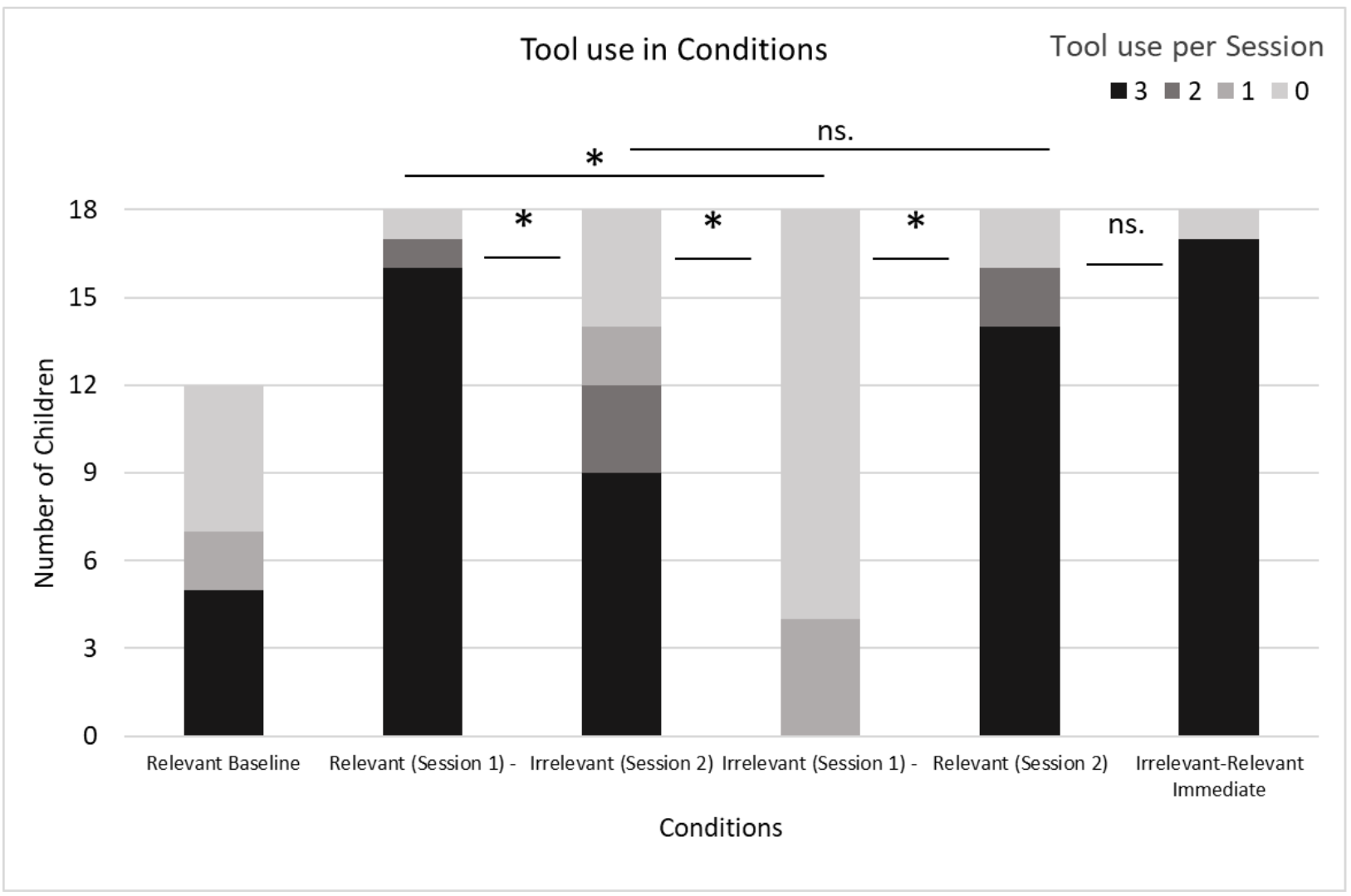

Figure 3. Tool use score (min. 0, max. 3) in Session 1 and Session 2, in the Relevant Baseline, Relevant-Irrelevant, Irrelevant-Relevant, and Irrelevant-Relevant Immediate conditions. Preschoolers either used a tool or reached for the object by hand. Asterisks indicate a significant difference ${ }^{*} p<.05$.

\section{Discussion}

In the present study 3-year-old children imitated a goal-directed action by considering the constraints of the context. In Session 1 the imitative strategy of the two experimental groups differed clearly in accordance with the situational constraints: if the tool was relevant for goal attainment, they used it, but when it was unnecessary, they omitted the tool use. This finding is similar to the pattern what was found with 2year-olds (Liszkai-Peres, Kampis et al., 2021), and in line with studies demonstrating the importance of efficiency evaluation in goal attainment during imitation (Bekkering 
Running Head: 3-year-old children's memory flexibility allows adaptation to an altered context

et al., 2000; Carpenter et al., 2005; Gergely et al., 2002). In accordance with the present finding, in the study of Fong et al. (2021) 4- to 6-year-old preschoolers chose the more efficient tool in a goal-attainment situation, even though the other less efficient but functionally working tool was supported by normative labels (e.g. "everybody uses this"). Based on these results, we provide further evidence that in the case of tools, instrumental and efficiency cues could have a stronger influence on behavior than social or normative cues (DiYanni \& Kelemen, 2008). When learning about instrumental functions, children seem to prioritize efficiency cues over social ones, and this results in less over-imitation in the learning phase. Overall, these results contribute to the larger picture of over-imitation being context dependent (Hoehl et al., 2019).

When the constraint changed from irrelevant to relevant immediately after demonstration, 3-year-old children used the tool almost at ceiling rate. This result further supports the claim that following a shorter interval, preschoolers show high behavioral flexibility in order to accommodate to a situation for successful goal attainment (Király et al., 2018; Scarf et al., 2013; Williamson et al., 2008). In the deferred imitation conditions, where one week passed between the first and second sessions, the behavior of the preschoolers was also adapted to the modified context, as indicated by the significant difference in rate of tool use between the two sessions in both conditions. Taken together, these results provide evidence that 3-yearchildrens's episodic memory capacities are developed enough to successfully reevaluate previously discarded action steps and incorporate them into their actions in a changed context.

At the same time, looking only at Session 2, the rate of tool use did not differ between conditions. This was mainly due to the fact that while in the Relevant- 
Running Head: 3-year-old children's memory flexibility allows adaptation to an altered context

Irrelevant condition children used the tool significantly less in the second session, they did not fully omit the tool use. Indeed, children were more flexible in their strategy change in the Irrelevant-Relevant condition - where children were able to recall the previously irrelevant means - and showed slightly more inflexible behavior in the Relevant-Irrelevant condition - where they were less prone to skip the unnecessary tool use. This result is in contrast to the findings with 2-year-olds in the same paradigm where an opposite tendency was observed: 2-year-olds easily omitted tool use within the new irrelevant constraints, but retrieval of a former irrelevant act caused more difficulty (Liszkai-Peres, Kampis et al., 2021).

The relatively inflexible behavior in the second session of the Relevant-Irrelevant condition could be explained by the fact that the constraint change in this condition does not motivate strategy change as much as in the Irrelevant-Relevant context, where the previous solution of the children did not work anymore. In fac, in the Relevant-Irrelevant condition the constraint change still allowed the use of the previously working means, only less efficiently. Preserving the already learned solution could indicate that in the long-term children associated the tool with the context as a functionally and socially accepted way of goal attainment taught by the model, and thus they tend not to question its relevance anymore (Kampis, Király, \& Topál, 2014). From preschool years onwards humans tend to stick to former working solutions and often do not question the reasons behind an act. This is illustrated in the anecdote of Sylvia's recipe, where the protagonist had always cut the end of the ham before putting it into the oven, just like she learned from her mother, only to find out later from the mother that she only did so because she did not have a large enough pan (Gergely \& Csibra, 2006). In this story, Sylvia re-enacted a successful observed strategy without 
Running Head: 3-year-old children's memory flexibility allows adaptation to an altered context

evaluating or questioning whether each step is necessary for the successful outcome (in the story, the delicious ham). In line with this, in the study of Carr, Kendal, \& Flynn (2015) efficacy of a goal reaching behavior was varied. They found that between the age of 4 to 9 years children copied even the least efficient behavior instead of innovating a new means (Carr et al., 2015). Moreover, learning about a tool is often part of cultural knowledge; its logic sometimes appears to be cognitively opaque, so high fidelity imitation is an adequate strategy and a quick solution for learning about such cultural artifacts (Casler \& Kelemen, 2005; Gardiner, Greif, \& Bjorklund, 2011; Gergely \& Csibra, 2006). Social factors like norms also strengthen the preservation of a previous means (Kenward, Karlsson, \& Persson, 2011), and could result in overimitation (Hoehl et al., 2019). While we did not observe over-imitation in the first session, as indicated by the difference in rate of tool use between irrelevant and relevant contexts in Session 1, it is plausible that once children re-enacted tool use as a successful strategy obtained in a communicative context, they stored it as part of the action sequence and did not re-evaluate its necessity in Session 2. These results highlight the interaction between children's memory capacities and the situational factors that trigger them: children in our study clearly seemed capable of flexibly reevaluating the necessity of action steps and adjusting their behavior, but their tendency to apply these skills was modulated by the context.

The main finding of the present study is the highly flexible behavior of 3-year-old children as indicated by the fact that they were able to retrieve a formerly irrelevant act at nearly ceiling level both after a short (several minutes) or long (one week) delay. This result is in line with Király et al. (2018), where 3-year-old children were able to revise their previous beliefs, if they got acquainted with new information influencing the 
Running Head: 3-year-old children's memory flexibility allows adaptation to an altered context

interpretation of a previous situation. The results also strengthen the findings of Williamson \& Meltzoff (2011), because children could easily apply an irrelevant step presented by a model, that became necessary in the test phase shortly after the demonstration. Moreover, the delay introduced in our study expands this kind of flexibility from short-term to long-term memory processes, as children were able to access former knowledge even after a week delay. In addition, flexibly manipulating elements of an event seems to be an easier task for 3-years-olds when done retrospectively as opposed to doing so in episodic foresight tasks (Scarf et al., 2013; Suddendorf et al., 2011). This retrospective manipulation might be a clearer index of episodic memory capacity as this task does not require the use of executive functions like planning as much.

Deferred imitation was the first method to explore the mnemonic capacity of nonverbal children (Jones \& Herbert, 2006; Meltzoff, 1988), and it is a behavioral indicator of adult memory as well (McDonough, Mandler, McKee, \& Squire, 1995). It is generally accepted that deferred imitation taps into declarative memory, the system responsible for storing knowledge and events that could be retrieved consciously later (Jones \& Herbert, 2006). However, it is highly debated whether deferred imitation could be an indicator of episodic memory as well, which is responsible for preserving onetime experiences in a detailed manner and allows re-experiencing earlier events (Tulving, 2005). As a complex phenomenon, it is difficult to access episodic memory on a behavioral level. However, with the flexibility task presented here we might get closer to it, as preservation of a long-term memory, including irrelevant elements, and the flexible reevaluation process are both clearly characteristics of episodic memory. Moreover, our findings point to the direction that memory processes behind imitation 
Running Head: 3-year-old children's memory flexibility allows adaptation to an altered context

might work on an inferential basis as the goal attainment event from a week earlier clearly interacted with the current situational constraints and efficacy cues. Flexible information access as part of episodic memory is supported by the inference-based account of Klein, Cosmides, Gangi, Jackson, \& Tooby (2009). They propose that a possible function of episodic memory is this kind of reevaluation process: the refinement of inferences originating form past experiences in order to make better predictions for the future.

In sum, 3-year-old children's memory shows some feauters of episodic memory, as they were flexible enough to retrieve and revise an irrelevant act, if it was necessary in a novel context. At the same time, they seemed to rely greatly on former experiences from a communicative demonstration, and if the context allowed for their previous solution to be used, they were less likely to deviate from it. In an earlier study, 2-yearolds were not able to fully incorporate a previously omitted action into their behavior. Together, these findings indicate that the behavior of children in the preschool years is increasingly facilitated by their developing memory capacities, and influenced by the context they are applied in. 
Running Head: 3-year-old children's memory flexibility allows adaptation to an altered context

\section{References}

Atance, C. M., \& Sommerville, J. A. (2014). Assessing the role of memory in preschoolers' performance on episodic foresight tasks. Memory, 22(1), 118-128. https://doi.org/10.1080/09658211.2013.820324

Bauer, P. J., \& Leventon, J. S. (2013). Memory for one-time experiences the second year of life: Implications for the status of episodic memory. Infancy, 18(5), 755781. https://doi.org/10.1111/infa.12005

Bekkering, H., Wohlschläger, A., \& Gattis, M. (2000). Imitation of gestures in children is goal-directed. Quarterly Journal of Experimental Psychology Section A: Human Experimental Psychology, 53(1), 153-164. https://doi.org/10.1080/713755872

Blankenship, T. L., \& Kibbe, M. M. (2019). Examining the limits of memory-guided planning in 3- and 4-year olds. Cognitive Development, 52(October), 100820. https://doi.org/10.1016/j.cogdev.2019.100820

Blankenship, T. L., \& Kibbe, M. M. (2022). Two-year-olds use past memories to accomplish novel goals. Journal of Experimental Child Psychology, 214, 105286. https://doi.org/10.1016/j.jecp.2021.105286

Brugger, A., Lariviere, L. A., Mumme, D. L., \& Bushnell, E. W. (2007). Doing the right thing: Infants' selection of actions to imitate from observed event sequences. Child Development, 78(3), 806-824. https://doi.org/10.1111/j.14678624.2007.01034.x

Carpenter, M., Call, J., \& Tomasello, M. (2005). Twelve- and 18-month-olds copy actions in terms of goals. Developmental Science, 8(1), F13-20. 
Running Head: 3-year-old children's memory flexibility allows adaptation to an altered context

https://doi.org/10.1111/j.1467-7687.2004.00385.x

Carr, K., Kendal, R. L., \& Flynn, E. G. (2015). Imitate or innovate? Children's innovation is influenced by the efficacy of observed behaviour. Cognition, 142, 322-332. https://doi.org/10.1016/j.cognition.2015.05.005

Casler, K., \& Kelemen, D. (2005). Young children's rapid learning about artifacts. Developmental Science, 8(6), 472-480. https://doi.org/10.1111/j.14677687.2005.00438.x

Csibra, G., \& Gergely, G. (2009). Natural pedagogy. Trends in Cognitive Sciences, 13(4), 148-153. https://doi.org/10.1016/j.tics.2009.01.005

DiYanni, C., \& Kelemen, D. (2008). Using a bad tool with good intention: Young children's imitation of adults' questionable choices. Journal of Experimental Child Psychology, 101(4), 241-261. https://doi.org/10.1016/j.jecp.2008.05.002

Fong, F. T. K., Imuta, K., Redshaw, J., \& Nielsen, M. (2021). When efficiency attenuates imitation in preschool children. British Journal of Developmental Psychology, 39(2), 330-337. https://doi.org/10.1111/bjdp.12366

Gardiner, A. K., Greif, M. L., \& Bjorklund, D. F. (2011). Guided by intention:

Preschoolers' imitation reflects inferences of causation. Journal of Cognition and Development, 12(3), 355-373. https://doi.org/10.1080/15248372.2010.542216

Gergely, G., Bekkering, H., \& Király, I. (2002). Developmental psychology: Rational imitation in preverbal infants. Nature, 415(6873), 755-755. https://doi.org/10.1038/415755a

Gergely, G., \& Csibra, G. (2003). Teleological reasoning in infancy: The naïve theory of rational action. Trends in Cognitive Sciences, 7(7), 287-292. 
Running Head: 3-year-old children's memory flexibility allows adaptation to an altered context

https://doi.org/10.1016/S1364-6613(03)00128-1

Gergely, G., \& Csibra, G. (2006). Sylvia's recipe: The role of imitation and pedagogy in the transmission of cultural knowledge. In N. J. Enfield \& S. C. Levenson (Eds.), Roots of Human Sociality: Culture, Cognition, and Human Interaction (pp. 229-255). Oxford: Berg Publishers.

Hoehl, S., Keupp, S., Schleihauf, H., McGuigan, N., Buttelmann, D., \& Whiten, A. (2019). 'Over-imitation': A review and appraisal of a decade of research. Developmental Review, 51(August 2018), 90-108. https://doi.org/10.1016/j.dr.2018.12.002

Jones, E. J. H., \& Herbert, J. S. (2006). Exploring memory in infancy: Deferred imitation and the development of declarative memory. Infant and Child Development, 15(2), 195-205. https://doi.org/10.1002/icd.436

Jones, S. S. (2009). The development of imitation in infancy. Philosophical Transactions of the Royal Society B: Biological Sciences, 364(1528), 23252335. https://doi.org/10.1098/rstb.2009.0045

Juvrud, J., \& Gredebäck, G. (2020). The teleological stance: Past, present, and future. Developmental Science, 23(5), 1-12. https://doi.org/10.1111/desc.12970

Kampis, D., Király, I., \& Topál, J. (2014). Fidelity To Cultural Knowledge and the Flexibility of Memory in Early childhood. In Naturalistic approaches to culture. Neurocognitive development and impairments (pp. 157-169). Budapest: Akadémiai Kiadó.

Kenward, B., Karlsson, M., \& Persson, J. (2011). Over-imitation is better explained by norm learning than by distorted causal learning. Proceedings of the Royal 
Running Head: 3-year-old children's memory flexibility allows adaptation to an altered context

Society B: Biological Sciences, 278(1709), 1239-1246.

https://doi.org/10.1098/rspb.2010.1399

Király, I. (2009). The effect of the model's presence and of negative evidence on infants' selective imitation. Journal of Experimental Child Psychology, 102(1), 14-25. https://doi.org/10.1016/j.jecp.2008.06.003

Király, I., Csibra, G., \& Gergely, G. (2013). Beyond rational imitation: Learning arbitrary means actions from communicative demonstrations. Journal of Experimental Child Psychology, 116(2), 471-486.

https://doi.org/10.1016/j.jecp.2012.12.003

Király, I., \& Oláh, K. (2020). Action selection in imitation: Why do we still need the teleological stance? Commentary on 'The teleological stance: Past, present, and future' by Juvrud and Gredeback. Developmental Science, 23(5), 10-12. https://doi.org/10.1111/desc. 12972

Király, I., Oláh, K., Csibra, G., \& Kovács, Á. M. (2018). Retrospective attribution of false beliefs in 3-year-old children. Proceedings of the National Academy of Sciences, 115(45), 11477-11482. https://doi.org/10.1073/pnas.1803505115

Klein, S. B., Cosmides, L., Gangi, C. E., Jackson, B., \& Tooby, J. (2009). Evolution and episodic memory: An analysis and demonstration of a social function of episodic recollection. Social Cognition, 27(2), 283-319.

https://doi.org/10.1521/soco.2009.27.2.283

Kline, M. A., Gervais, M. M., Moya, C., \& Boyd, R. T. (2020). Irrelevant-action imitation is short-term and contextual: Evidence from two under-studied populations. Developmental Science, 23(3). https://doi.org/10.1111/desc.12903 
Running Head: 3-year-old children's memory flexibility allows adaptation to an altered context

Liszkai-Peres, K., Kampis, D., \& Király, I. (2021). The flexibility of early memories: Limited reevaluation of action steps in 2-year-old infants. Journal of Experimental Child Psychology, 203, 105046. https://doi.org/10.1016/j.jecp.2020.105046

Marsh, L. E., Ropar, D., \& Hamilton, A. F. d. C. (2019). Are you watching me? The role of audience and object novelty in overimitation. Journal of Experimental Child Psychology, 180, 123-130. https://doi.org/10.1016/j.jecp.2018.12.010

McDonough, L., Mandler, J. M., McKee, R. D., \& Squire, L. R. (1995). The deferred imitation task as a nonverbal measure of declarative memory. Proceedings of the National Academy of Sciences of the United States of America, 92(16), 7580-7584. https://doi.org/10.1073/pnas.92.16.7580

Meltzoff, A. N. (1988). Infant Imitation After a 1-Week Delay: Long-Term Memory for Novel Acts and Multiple Stimuli. Developmental Psychology, 24(4), 470-476. https://doi.org/10.1037/0012-1649.24.4.470

Meltzoff, A. N. (1995a). Understanding the Intentions of Others: Re-Enactment of Intended Acts by 18-Month-Old Children. Developmental Psychology, 31(5), 838-850. https://doi.org/10.1037/0012-1649.31.5.838

Meltzoff, A. N. (1995b). What infant memory tells us about infantile amnesia: Longterm recall and deferred imitation. Journal of Experimental Child Psychology, 59(3), 497-515. https://doi.org/10.1006/jecp.1995.1023

Meltzoff, A. N., \& Marshall, P. J. (2018). Human infant imitation as a social survival circuit. Current Opinion in Behavioral Sciences, 24(Figure 1), 130-136. https://doi.org/10.1016/j.cobeha.2018.09.006

Meltzoff, A. N., \& Moore, M. K. (1983). Newborn infants imitate adult facial gestures. 
Running Head: 3-year-old children's memory flexibility allows adaptation to an altered context

Child Development, 54(3), 702-709. https://doi.org/10.1111/j.1467-

8624.1983.tb00496.x

Meltzoff, A. N., \& Williamson, R. A. (2013). Imitation: Social, cognitive, and theoretical perspectives. The Oxford Handbook of Developmental Psychology: Volume 1 Body and Mind, 1, 651-682.

Nielsen, M. (2006). Copying actions and copying outcomes: Social learning through the second year. Developmental Psychology, 42(3), 555-565. https://doi.org/10.1037/0012-1649.42.3.555

Nielsen, M., \& Blank, C. (2011). Imitation in Young Children: When Who Gets Copied Is More Important Than What Gets Copied. Developmental Psychology, 47(4), 1050-1053. https://doi.org/10.1037/a0023866

Over, H., \& Carpenter, M. (2012). Putting the social into social learning: Explaining both selectivity and fidelity in children's copying behavior. Journal of Comparative Psychology, 126(2), 182-192. https://doi.org/10.1037/a0024555

Paulus, M. (2014). How and why do infants imitate? An ideomotor approach to social and imitative learning in infancy (and beyond). Psychonomic Bulletin and Review, 21(5), 1139-1156. https://doi.org/10.3758/s13423-014-0598-1

Ray, E., \& Heyes, C. (2011). Imitation in infancy: The wealth of the stimulus. Developmental Science, 14(1), 92-105. https://doi.org/10.1111/j.14677687.2010.00961.x

Scarf, D., Gross, J., Colombo, M., \& Hayne, H. (2013). To have and to hold: Episodic memory in 3- and 4-year-old children. Developmental Psychobiology, 55(2), 125-132. https://doi.org/10.1002/dev.21004 
Running Head: 3-year-old children's memory flexibility allows adaptation to an altered context

Simpson, A., \& Riggs, K. J. (2011). Three- and 4-year-olds encode modeled actions in two ways leading to immediate imitation and delayed emulation.

Developmental Psychology, 47(3), 834-840. https://doi.org/10.1037/a0023270

Stengelin, R., Hepach, R., \& Haun, D. B. M. (2019). Being observed increases overimitation in three diverse cultures. Developmental Psychology, 55(12), 2630-2636. https://doi.org/10.1037/dev0000832

Suddendorf, T., Nielsen, M., \& Von Gehlen, R. (2011). Children's capacity to remember a novel problem and to secure its future solution. Developmental Science, 14(1), 26-33. https://doi.org/10.1111/j.1467-7687.2010.00950.x

Tulving, E. (2005). Episodic memory and autonoesis: Uniquely human. In H. S. Terrace \& J. Metcalfe (Eds.), The Missing Link in Cognition: Origins of selfreflective consciousness (pp. 4-56). New York: Oxford University Press. https://doi.org/10.1093/acprof:oso/9780195161564.003.0001

Want, S. C., \& Harris, P. L. (2001). Learning from other people's mistakes: Causal understanding in learning to use a tool. Child Development, 72(2), 431-443. https://doi.org/10.1111/1467-8624.00288

Williamson, R. A., \& Meltzoff, A. N. (2011). Own and others' prior experiences influence children's imitation of causal acts. Cognitive Development, 26(3), 260268. https://doi.org/10.1016/j.cogdev.2011.04.002

Williamson, R. A., Meltzoff, A. N., \& Markman, E. M. (2008). Prior Experiences and Perceived Efficacy Influence 3-Year-Olds' Imitation. Developmental Psychology, 44(1), 275-285. https://doi.org/10.1037/0012-1649.44.1.275 